\title{
Editorial
}

\section{Toward Better Antibiotic Use in Hospitals}

\author{
Stephen B. Kritchevsky, PhD; Bryan P. Simmons, MD
}

See page 710 .

In order to promote hospitalwide quality improvement, the Joint Commission on Accreditation of Healthcare Organizations is asking hospitals to break down departmental barriers and focus on aspects of care that cross departmental lines. The article by Lee et al $^{1}$ describes the initial experience of one hospital in examining proper antibiotic usage from a crossdepartmental perspective, combining the data and expertise of clinical pharmacy, microbiology, and the infectious disease service.

A comprehensive evaluation of antibiotic use would include every aspect of the process, including 1) the decision to prescribe antibiotics to a patient, 2) initial antibiotic selection, 3) monitoring patient response, 4) monitoring for drug toxicity or adverse reactions, 5) the decision to obtain cultures, 6) the physician response to culture/susceptibility information, and 7) the termination of therapy The evaluation described by Lee et al enters the process at the point where culture and susceptibility results become available. In part, the decision to begin the evaluation at this point is dictated by the purviews of the departments collaborating in the project, but two additional considerations come into play.

First, susceptibility results are data that provide a basis for rational action. With susceptibility results at hand, it is indubitable that giving an antibiotic to which the infecting organism is resistant is wrong, and that the giving of multiple or broad-spectrum antibiotics, where fewer or more specific ones would do, is not cost-effective.
The second advantage of the approach of Lee et al is that the microbiology and clinical pharmacy departments represent points of data concentration. If the only way to get information about susceptibilities and prescribed antibiotics were to go to the patient record, this project would be labor intensive and expensive to execute. The microbiology susceptibility reports represent a major concentration of relevant patient data, justifying the investigators' efforts to link these data manually to the computerized pharmacy database. Several institutions already have automated the link between microbiology and pharmacy to permit the efficient identification of patients who may benefit from an alteration in their antibiotic regimens. ${ }^{2-4}$ Other automated linkages have been described, including linking the laboratory and pharmacy databases to identify patients with impaired renal function who are receiving nephrotoxic drugs and to evaluate whether drug levels are being monitored in patients who require it. ${ }^{2}$ The availability of a computerized medical record is a prerequisite if the comprehensive and routine monitoring of all aspects of antibiotic use is to be affordable.

The fact that Lee et al sampled reports from 1 week per month has strengths and weaknesses depending upon one's point of view. From a traditional quality assurance standpoint, the sampling might be considered inadequate because a full 40 weeks per year were not evaluated. The attitude of traditional quality assurance is that physicians will do a better job of prescribing if they are slapped on their wrists every time they

From the Department of Biostatistics and Epidemiology, University of Tennessee, Memphis, Tennessee ( $D r$. Kritchevsky), and Methodist Hospitals of Memphis, Memphis, Tennessee (Dr. Simmons).

Address reprint requests to Stephen B. Kritchevsky, $P h D$, Department of Biostatistics and Epidemiology, University of Tennessee, 877 Madison Ave, Memphis, TN 38163. 690.

94-ED-106. Kritchevsky SB, Simmons BP Toward better antibiotic use in hospitals. Infect Control Hosp Epidemiol 1994;15:688- 
are found doing something wrong. It has become increasingly evident that this punitive approach fails to improve quality because it fails to recognize the true reasons for poor quality. Most failures to reach a desired level of quality are not the result of individuals acting badly, but rather because the system in which the workers are functioning is designed in such a way as to make achieving high quality difficult. ${ }^{5}$ Antibiotics are misused, not because physicians are willfully negligent, but because the system in which the physicians are operating makes it difficult for them to do the right thing.

In a quality improvement system based on the premises of continuous quality improvement, the sampling strategy of Lee et al is entirely appropriate and satisfactory. A one twelfth sample supplies enough data to serve the two major purposes of data gathering in a continuous quality improvement program: it provides a base against which to measure future improvement, and it documents the relative importance of differing quality problems. This allows the targeting of quality improvement efforts at aspects of the process that will do the most good. In their example, Lee et al found that $24(49 \%)$ of the 49 variances were attributable to the failure to switch to a cheaper antibiotic or the failure to change therapy in light of susceptibility information. That these variances were the result of a weakness in the process and not the bad behavior of a few physicians is suggested by the authors' observation that the variances they identified did not cluster by either individual or department.

Berwick et $\mathrm{al}^{6}$ wrote of two journeys in quality improvement, a diagnostic journey and a remedial journey. The project described by Lee et al represents the former; but now that we have identified the most common problems with antibiotic use, what next? To understand what to do next, one must understand the root causes of the problems.

Evidence shows that many failures of physicians to use medical resources optimally represent failures in attention as much as anything else. ${ }^{7} \mathrm{~A}$ tremendous volume of information about patients and patient care competes for the physician's attention. Information that directly relates to saving lives and improving health must command the best of a physician's attention. The attention required to tailor care to make it more efficient simply may not be available in the face of more pressing and immediate patient care concerns. If healthcare organizations want to promote more efficient care, they must supply an information infrastructure to support it. In an outpatient health management organization setting, the prevalence of untreated patients with a positive streptococcus test fell from over $10 \%$ to $3 \%$ after a computerized reminder system was put into effect. ${ }^{8}$ The rate immediately rebounded after the reminder system was terminated, suggesting that most of the failures were attributable to the difficulty of physicians in keeping up with all of the patients needing follow-up.

Similarly, Schentag et $\mathrm{a}^{4}$ described an active clinical pharmacy program aimed at improving the use of antimicrobials at a 450-bed hospital. In their program, the susceptibility reports automatically are linked to the pharmacy database, and a computerized expert system identifies patients who might benefit from intervention by a clinical pharmacist. The system addresses exactly those quality deficiencies that Lee et al found to be most common in their hospital, namely the failure to use the least expensive antibiotic appropriate for the infection and the failure to switch to the appropriate antibiotic given the susceptibility findings. In the experience of Schentag et al, 'Virtually all intervention proposals made to the physicians were accepted, but in situations where we did not contact physicians, therapy of virtually identical patients proceeded with the expensive, empirically chosen regimen in almost all cases."

Active programs, such as that described by Schentag et $a l,{ }^{4}$ depend upon the frequent interaction between physicians and "inspectors" (in this case, a clinical pharmacist). The theory underlying continuous quality improvement holds that this reliance on inspection for quality is undesirable. Only recently, however, has the technology existed to replace such inspection-based systems effectively. Bedside computers, in conjunction with expert system software, provide the opportunity to guide the physicians decision-making process at the point of care. The physician can consider the computer recommendations with all the pertinent information available to him. The advantages of such systems are that they prevent prescribing errors before they occur, they can automatically log "variances" for further review, and they eliminate waste both in terms of the money associated with suboptimal prescribing and the time associated with identifying and correcting prescription errors. Other suggestions to improve the use of antibiotics have included changing antibiotic ordering forms to emphasize the correct dosing, excluding drugs from the hospital formulary, requiring special justification for the use of very expensive drugs, requiring consultation with the infectious disease department before ordering certain antibiotics, and the use of automatic stop orders to terminate use of certain drugs. ${ }^{9,10}$ Some have been shown to be effective; others have not. When knowledge deficits are the problem, by far the most effective programs have involved the face-to-face encounter strategies between physicians and a knowledgeable advocate. This strat- 
egy, sometimes called "academic detailing," repeatedly has been shown to be effective in changing physicians' prescribing patterns. ${ }^{11,12}$

The project described by Lee et al is a first and necessary step in a valuable quality improvement effort. It provides the data foundation for future improvement. Their strategy of sending informational letters to physicians found at variance probably will not result in a great deal of improvement, since the majority of the variances represent failures of the system and not the purposeful misconduct or ignorance of the physician. If this endeavor is approached in the spirit of continuous improvement, the improvement process will involve representatives of the medical staff, nursing, and information systems, in addition to microbiology, clinical pharmacy, and infectious disease. The collaboration of microbiology and clinical pharmacy can describe what goes wrong and how often it goes wrong, but wider input will be necessary to determine why things go wrong and what to do about it.

\section{REFERENCES}

1. Lee KR, Leggiadro RJ, Burch KJ. Drug use evaluation of antibiotics in a pediatric teaching hospital. infect Control Hosp Epidemiol 1994;15:710-712.

2. Burke JP, Classen DC, Pestotnik SL, Evans RS, Stevens LE. The
HELP system and its application to infection control. $J$ Hosp Infect 1991;18(suppl A):424-431.

3. Scarafile PD, Campbell BD, Kilroy JE, Mathewson HO. Computerassisted concurrent antibiotic review in a community hospital. Am J Hosp Pharm 1985;42:313-315.

4. Schentag JJ, Ballow CH, Fritz AL, et al. Changes in antimicrobial agent usage resulting from interactions among clinical pharmacy, the infectious disease division, and the microbiology laboratory. Diagn Microbiol Infect Dis 1993;16:255-264.

5. Kritchevsky SB, Simmons BE Continuous quality improvement: concepts and applications for physician care. JAMA 1991;266:18171823.

6. Berwick DM, Godfrey AB, Roessner J. Curing Health Care: New Strategies for Quality Improvement. San Francisco, CA: JosseyBass Inc; 1990.

7. Soumerai SB, McLaughlin TJ, Avorn J. Improving drug prescribing in primary care: a critical analysis of the experimental literature. Milbank $Q 1989 ; 67: 268-317$.

8. Bamett GO, Winickoff RN, Dorsey JL, Morgan MM, Lurie RS. Quality assurance through automated monitoring and concurrent feedback using a computer-based medical information system. Medical Care 1978;16:962-970.

9. Kunin CM. Problems of antibiotic usage: definitions, causes and proposed solutions. Ann Intern Med 1978;89:802-805.

10. Avorn J, Soumerai SB, Taylor W, Wessels MR, Janousek J, Weiner M. Reduction of incorrect antibiotic dosing through a structured educational order form. JAMA 1988;148:1720-1724.

11. Soumerai SB, Avom J. Principles of educational outreach ("academic detailing") to improve clinical decision making. JAMA 1990:263:549-556.

12. Schaffner W, Ray WA, Federspiel CF, Miller WO. Improving antibiotic prescribing in office practice: a controlled trial of three educational methods. JAMA 1983;250:1728-1732.

\section{NIOSH Accused of Concealing Respirator Deficiencies}

\section{by Gina Pugliese, RN, MS Medical News Editor}

The National Institute for Occupational Safety and Health (NIOSH) is reviewing allegations of a former senior NIOSH official, Nelson A. Leidel, that respirator deficiencies were concealed during the respirator certification process. In a lengthy document from Leidel to NIOSH Director Linda A. Rosenstock, Leidel asserted that NIOSH has engaged in questionable practices related to certification of respirators over a 22-year period that included use of certification criteria that do not assess respirator performance adequately. Leidel retired from NIOSH on September 1, 1994, after more than 20 years with the job safety and health institute. From 1987 to 1992 he managed the project to revise the respirator certification requirements.

A NIOSH spokesman said the institute is convening an internal task force to study the 75 page critique of NIOSH's certification procedures from
Leidel, which has been included in the rulemaking docket for NIOSH's recent proposal for revising its certification procedures. NIOSH Director Rosenstock said that the issues raised by Leidel were complex and required some study and that NIOSH will issue a detailed response.

FROM: Bureau of National Affairs Daily Reporter System: Daily Labor Report (No. 180; September 20, 1994:A8-A11 and No. 186; September 28, 1994:A16). 